\title{
Oxygen-ozone therapy: an extra weapon for the general practitioners and their patients
}

\author{
Nicolò Allorto \\ Italian Society for Oxygen-Ozone Therapy (SIOOT), Gorle (BG), Italy
}

\begin{abstract}
Oxygen-ozone therapy is a medical practice validated in the literature by numerous international clinical studies. It has indications in many diseases and complications, alone or in combination with conventional therapies. In this work, a number of international clinical studies have been evaluated and those most relevant to pathologies of daily interest to the general practitioner (GP) have been selected to assess their cost/benefit ratio. It has been concluded that GPs and their patients can derive significant benefits from this medical practice, and GPs are ideally placed to assess which patients should receive this treatment.
\end{abstract}

\section{Introduction}

Ozone $\left(\mathrm{O}_{3}\right)$ is an allotropic form of oxygen and its molecules are formed by three atoms of oxygen.

At room temperature it is an unstable gas and is present in nature in the atmosphere and in the ambient air in small concentrations.

Ozone was discovered in the early decades of the nineteenth century by a German chemist, naturalized Swiss, Christian Friedrich Schonbein during experiments of slow oxidation of white phosphorus and electrolysis of water.

Correspondence: Nicolò Allorto, Italian Society for Oxygen-Ozone Therapy (SIOOT), via Don Luigi Sturzo 2, 24020 Gorle (BG), Italy. E-mail: nicallorto@gmail.com

Key words: Ozone; General practitioners; Antioxidant system.

Conflict of interest: the author declares no potential conflict of interest.

Funding: none.

Received for publication: 25 July 2019.

Accepted for publication: 29 July 2019.

${ }^{\circ}$ Copyright: the Author(s), 2019

Licensee PAGEPress, Italy

Ozone Therapy 2019; 4:8424

doi:10.4081/ozone.2019.8424

This article is distributed under the terms of the Creative Commons Attribution Noncommercial License (by-nc 4.0) which permits any noncommercial use, distribution, and reproduction in any medium, provided the original author(s) and source are credited.
Its use in medicine dates back to 1915 in Germany where, during the Great War, the bactericidal effect of the ozone was observed: it was an intuition of Dr. Hans Wolff who used it on infected wounds treating thousands of soldiers suffering from gaseous gangrene.

Today, ozone therapy is a medical practice used throughout the world: in India and China it is used mainly for the treatment of herniated discs and associated syndromes, ${ }^{1-9}$ in Cuba it has been used since the sixties in many hospitals to make up for the lack of numerous conventional drugs due to the U.S. embargo, and in many European countries there are specializations and university masters.

The use of this gaseous mixture for therapeutic purposes in Italy dates back to 1983 with the establishment of the Italian Society for Oxygen-Ozone Therapy (SIOOT) which has established rules and therapeutic protocols that ozone therapy doctors must follow in accordance with the Higher Institute of Health.

In some Italian regions, including Lombardy, the Ozone Oxygen Therapy is included in the Therapeutic Handbook for the treatment of vascular ulcers associated with venous insufficiency. In the literature there are over 2000 scientific papers published internationally that certify its validity as a complementary medical practice in many inflammatory, infectious, degenerative diseases and in rehabilitation after acute ischemic cardiac and cerebral events.

\section{Mechanisms of action}

As already mentioned, ozone is an allotropic form of oxygen consisting of three of it, it is an unstable gas so it can neither be stored nor transported but must be produced by special machinery called ozonizers. This equipment draws medical oxygen from the cylinders and converts oxygen into ozone at the concentrations required by the doctor.

It exists in nature in the stratosphere where it forms the layer that absorbs most of the Sun's ultraviolet rays (UV-C) while in the troposphere it is formed in a transitory way when lightning, sparks, electrical discharges strike the oxygen present in the same.

Although not a radical, because it has an even number of electrons in the outer orbit, it is an energetic oxidizer and at high concentrations is toxic to the mucous membranes of the airways for its lipolytic properties; it is considered one of the environmental pollutants of urban areas measured together with the PM10 of the control units of air purity.

It is considered a powerful oxidant with the following characteristics:

- It has intrinsic antimicrobial activity (antibacterial, antiviral, antifungal and antiparasitic) and is able to strengthen the nonspecific immune defences of skin and mucous membranes by helping macrophages and polymorphonucleates (also using ROS oxygen radicals - as antimicrobials); 
- It determines the lipolysis of unsaturated organic substances (PUFA, which is a mechanism that can be exploited for the discolysis in herniated discs and in the treatment of cellulite;

- It stimulates the intra- and extra-cellular antioxidant system, generating transient oxidative stress, thus protecting the body's cells from the free radicals typical of inflammatory and degenerative diseases;

- It improves the microcirculation: partially and temporarily oxidizing the intra-erythrocyte GSH (Reduced Glutathione), it improves the membrane oxygenation of the red cell, increasing its flexibility and permeability; it reduces and modulates the inflammation and the immune response: oxidizing the lipidic polyunsaturated substances it intervenes in the inflammatory cascade of the eicosanoids (prostaglandins, leukotrienes and thromboxanes). ${ }^{10-14}$

\section{Clinical indications}

Ozone therapy as a medical practice can be used in many medical branches and for various diseases:

- Dermatology (herpes, zoster/simplex, acne, eczema, lipodystrophy, cellulitis, etc.);

- Internal medicine (atherosclerosis, autoimmune diseases, vascular complications of diabetes, scleroderma, etc.); $;^{15-21}$

- Cardiology (post-ischemia rehabilitation); $10,12,15$

- Orthopaedics (hernia and disc protrusions, arthritis, etc.) ${ }^{17}$

- Dentistry (caries, post-implant disinfection, canker sores, etc.);

- Vascular (complications of venous and arterial insufficiency, ulcers, sores, diabetic foot, etc. $){ }^{18}$

- Gastroenterology (intestinal dysbiosis, chronic intestinal inflammatory diseases, Helicobacter pylori gastritis, etc.);

- Physiatrics (tenosynovitis, chronic pain, low back pain, etc.);

- Surgery (infectious complications before and after surgery, treatment of difficult wounds, etc.); ${ }^{11,16,20}$

- Gynecology (mucous infections, etc.);

- Neurology (headache, adjuvant in multiple sclerosis, etc.);

- Rheumatology (rheumatoid arthritis, fibromyalgia, etc. $)^{19}$

\section{Contraindications and side effects}

The contraindications and side effects are not many but, as in all therapies and clinical practices, they must be known.

Ozone therapy is contraindicated in cases of: i) pregnancy, for lack of studies on the subject; ii) clinically manifested hyperthyroidism, for its effects on metabolism; iii) favism, for the greater susceptibility of the red cells to oxidative stress (only self-emoinfusion is contraindicated); iv) as collateral effect it is necessary to mention the toxicity that the ozone therapy exerts on the mucous membranes of the respiratory tract; v) as it has a lipolytic effect, the ozone therapy can affect the lung surfactant and must therefore be handled in closed circuits (not in PVC) and/or with adequate precautions.

\section{Methods of administration}

The administration methods are multiple and vary depending on the branch of medicine concerned, the pathology to be treated and the characteristics of the patient following the various protocols by the SIOOT.

\section{Essential requirements of clinical practice}

Since this is a medical practice, it is necessary, first of all, to have a degree in Medicine and Surgery and to be registered with the Order of Doctors. Also required: i) a theoretical and practical course or a University Master (Rome or Pavia) with regular annual updates according to SIOOT protocols; ii) the use of certified equipment (Decree 46/97 CEE93/42 in class 2A) with photometer, a critical instrument for the accurate production of the necessary ozone; iii) the use of therapeutic protocols and guidelines established by SIOOT; iv) medical practices equipped with life-saving drugs and devices.

\section{Objectives}

The objective of this work is to analyze some international scientific studies concerning the medical practice of oxygen-ozone therapy in the field of diseases affecting the general practitioner (GP) to assess the therapeutic potential in combination or as an alternative to conventional therapies.

\section{Search methods}

In the literature there are thousands of international articles related to the medical practice of oxygen-ozone therapy.

Those most relevant to the work of the GP were considered. Seven of these have been chosen, of which the abstract is reported and commented.

Intramuscolar oxigen-ozone therapy in the treatment of acute back pain with lumbar disc herniation: a multicentre, randomized, double-blind clinical trial of active and simulated lumbar paravertebral injection by Paoloni et al. ${ }^{1}$

Abstract: Study design: Multicenter randomized, double-blind, simulated therapy-controlled trial in a cohort of patients with acute low back pain (LBP) due to lumbar disc herniation (LDH).

Objective: To assess the benefit of intramuscular-paravertebral injections of an oxygen-ozone (O2O3) mixture.

Summary of background data: Recent findings have shown that $\mathrm{O} 2 \mathrm{O} 3$ therapy can be used to treat LDH that fails to respond to conservative management. However, these findings are based on intradiscal/intraforaminal $\mathrm{O} 2 \mathrm{O} 3$ injection, whereas intramuscular-paravertebral injection is the technique used most in clinical practice in Italy and other Western countries.

Methods: Sixty patients suffering from acute LBP caused by $L D H$ was randomized to an intramuscular $O 2 O 3$ or control group. Patients were observed up to assess pain intensity, LBP-related disability, and drug intake (15 [V2] and 30 [V3] days after treatment started, and 2 weeks [V4], and 3 [V5] and 6 [V6] months after treatment ended).

Results: A significant difference between the 2 groups in the percentage of cases who had become pain-free $(61 \%$ vs $33 \%$, $P<0.05)$ was observed at V6. Patients who received $O 2 O 3$ had a lower mean pain score than patients who received simulated therapy throughout the observation period. A significant improvement was observed in LBP-related disability in the study group patients when compared with the control group patients. Active $\mathrm{O} 2 \mathrm{O} 3$ therapy was followed by a significantly lower number of days on nonsteroidal anti-inflammatory drugs at V2 and 
$V 3$ and by a lower number of days at V4. No adverse events were reported.

Conclusion: Treatment of LBP and sciatica is a major concern. Although the natural history of acute LBP is often self-limiting, conservative therapies are not always effective; in such cases, 0203 intramuscular lumbar paravertebral injections, which are minimally invasive, seem to safely and effectively relieve pain, as well as reduce both disability and the intake of analgesic drugs.

Seventy patients with acute low back pain from herniated discs, diagnosed by imaging, were randomized between those who underwent intramuscular paravertebral injections of a mixture of Oxygen-Ozone ( 3 infiltrations per week for 5 consecutive weeks with $20 \mathrm{~mL}$ of a mixture of Oxygen-Ozone at the concentration of $20 \mathrm{mcg} / \mathrm{mL}$ ) and the control group. These patients were evaluated for pain intensity (VAS scale), degree of disability and need to take non-steroidal anti-inflammatory drugs (NSAIDs) or steroids after the start of treatment, after 15 days and 30 days, after 3 and 6 months. The study showed significant difference between the two groups $(61 \%$ vs $33 \%, \mathrm{P}<0.05)$ at 6 months after the end of treatment; those who received oxygen-ozone therapy reported less pain, less disability, less need to take doses of NSAIDs and steroids. This study demonstrated the effectiveness of the therapy, the very low invasiveness of the practice and the harmlessness of the method.

Usually, many patients with low back sciatica, cruralgia and neuralgia of various kinds - many of which depending on disc hernias - go to the GP's consulting room. The intramuscular para-vertebral infiltration of a mixture of oxygen-ozone allows considerable clinical benefit with savings of drugs burdened with significant complications (NSAIDs and steroids are known to be detrimental to the gastric mucosa, reduce renal function, promote osteoporosis, etc.).

Unfortunately, this practice is not included in the current Essential Levels of Care so the cost of the therapy is borne by the patient.

Important clinical result with savings of drugs, ${ }^{13}$ sometimes more harmful than effective, should be weighed against the cost of treatment and recommended to selected patients.

- Ozone therapy in gastroduodenal pathology associated with Helicobacter pylori, a multicentre, randomised, double-blind study by Fedorov et al. ${ }^{2}$

Abstract: Three methods of ozone therapy (intravenous injection of ozonized physiologic saline, oral intake of ozonized lowmineral water and combination of the two treatments) were compared by efficacy in 215 patients with gastroduodenal pathology associated with Helicobacter pilori. All the techniques of ozone therapy proved effective in relation to regress of clinical symptoms, regeneration processes, eradication of Helicobacter pylori, but the combined regimen was the best.

Three different oxygen-ozone therapy methods were tested in two hundred and fifteen patients with $H$. pylori gastritis: autohemoinfusion, oral administration of ozonized mineral water and the combination of both methods. All three techniques have been proved to be valid for the clinical management of symptoms and the eradication of the bacterium. The best results were obtained by combining both methods.

In the literature there is also an Italian pilot study by Dr. Sanna (FIMMG-METIS Research Department), in the setting of General Medicine. This study is carried out on a few patients of two GPs, one from Veneto and one from Sardinia, with proven diagnosis of gastritis by $H$. pylori by esophagogastroduodenoscopy or Urea Breath Test (UBT). They were given ozonized water at a dose of four glasses $(200 \mathrm{cc})$ per day for two weeks, after which eradication control was carried out. The results indicate that about $50 \%$ of patients have obtained complete inheritance with negative UBT or Fecal Ag, without drug therapy. The study is very interesting, but having been carried out on a small number of patients (seven), its statistical validity is low.

With an ozonator and more patients available, a larger study could be carried out and greater statistical validity achieved. If the data were confirmed, the number of patients to be treated in triple or quadruple therapy could be significantly reduced, thus reducing the cost for the National Health System as the cost would be negligible and borne by the patient. This could also mean less discomfort for patients (standard eradication therapies are based on combinations of high dosages of antibiotics, proton pump inhibitors and adjuvants that often create dyspepsia, diarrhea and other side effects) as well as reduction of antibiotic resistance.

- New method of treatment of pyoinfiammatory soft tissue complications in patients with diabetes mellitus, Ukrainian study by Karatieieva et $a l^{3}$

Abstract: Our study evaluated the levels of peroxide oxidation of lipids, oxidative modification of proteins, antioxidant protection and dynamic changes in markers of toxicity in patients with diabetes mellitus and purulent-inflammatory complications. In total, 124 patients were enrolled in the study and were divided into two groups according the treatment methods. Study group consisted of 53 patients, who received intravenously ozonized saline in addition to conservative treatment. The control group consisted of 71 patients who received only conventional therapy. The study period was 6-15 days. The results showed that the use of ozone therapy is not accompanied by negative injury responses at the level of hemostasis parameters and blood biochemical characteristics. Furthermore, ozone therapy may have a favorable effect on treatment outcome in patients with purulent-inflammatory complications and daibetes mellitus.

This study evaluated lipid peroxygenation levels, protein oxidation and modification, antioxidant protection and dynamic changes in inflammation and toxicity markers in patients with diabetes mellitus with inflammatory and/or purulent complications. The study enrolled one hundred and twenty-four patients divided into two groups: 53 of them received autoemoinfusion in addition to conventional treatments, while seventy-one only standard treatments. For both groups of these therapies were performed for 2 weeks. Patients undergoing both therapies showed clinical improvement in inflammatory complications, without rheological alterations or in the biochemical parameters evaluated.

This study confirmed the harmlessness of oxygen ozone therapy even in its most invasive form, i.e. the auto-hemoinfusion, assessing the stability of lipid peroxidation, protein and inflammatory markers, also showed improvements in symptoms and in the management of classical complications of diabetes.

A GP has numerous patients suffering from diabetes mellitus and its complications or other chronic inflammatory diseases that could benefit from the anti-inflammatory-immunomudulated effect of ozone, and a GP may recommend to selected patients the SIOOT oxygen-ozone therapy.

- Thermography improves clinical assessment in patients with systemic sclerosis treated with ozone therapy, a Polish study by Nowicka. $^{4}$

Abstract: Objective. Treatment of scleroderma is challenging and limited. The aim of our study was to evaluate the usefulness of thermography in assessment of the clinical condition (joints movability and skin thickness) in clinically advanced patients with systemic sclerosis before and after ozone therapy. Method. The study 
included 42 patients aged 32 to 73 years with advanced systemic sclerosis hospitalized in the university clinic between 2003 and 2006. Thermography and clinical examinations were conducted at baseline and after two series of bath in water with ozone. Results. The comparison of results showed significant increase in skin temperature by $2.5^{\circ} \mathrm{C}$, significant increase in interphalangeal joints movability by 18 degrees, and significant decrease in skin score by 14.7 points. The skin temperature was correlated with skin score $(r=-0.59)$ and joints movability $(r=+0.8)$. Conclusions. Ozone therapy shows positive effect on clinical parameters and skin temperature as measured with thermography. The study indicated possibility of introducing ozonotherapy as an independent therapy in cases with low level of progression or during remission periods and as additional treatment in patients with advanced disease requiring immunosuppressive treatment. Thermography is useful in assessment of skin condition showing strong correlation between skin temperature and clinical parameters.

In this study, 42 patients aged 32-73 years with advanced systemic sclerosis, mobility and articular rigidity and differences in thermography (used as an indicator of the characteristically reduced skin perfusion status in patients with systemic sclerosis) were evaluated before or after two sets of baths in tubs with ozonized water.

The results reported a significant increase in thermography $\left(+2.5^{\circ} \mathrm{C}\right)$ and joint mobility in the interphalangeal region, demonstrating how ozonized water can help to improve objective and subjective clinical parameters.

Ozone therapy can be used independently of other therapies during periods of disease remission and in association with immunosuppressive-immunomodulating therapies during active disease phases.

This study showed how a rather simple method could be of great help in a complex disease, multi-systemic and normally treated with large doses of steroidal anti-inflammatory drugs and/or immunosuppressant-immunomodulating limiting side effects of classical drugs and improving the subjective well-being of the patient.

Usualy, a GP does not have many patients with systemic sclerosis, as it is a rare disease, but this study can give an idea of how this clinical practice can be used and studied in many inflammatory systemic diseases with objective and subjective benefit and reduction of drug therapy. Moreover, the use of ozonized water is one of the cheapest and easiest ways to administer ozone.

- Beneficial effects of ozone therapy on oxidative stress, cardiac functions and clinical findings in patients with heart failure reduced ejection fraction, a Turkish study by Buyuklu et $a .^{5}$

Abstract: The aim of study was to determine the effects of ozone therapy on the oxidative stress, cardiac functions and clinical findings in patients with heart failure reduced ejection fraction (HFrEF). A total of 40 patients with New York Heart Association 2 and $3 \mathrm{HF}$ with left ventricular ejection fraction $(L V E F)<35 \%$, and 40 subjects without HF as control group were included in the study. Patients with HFrEF were given additional ozone therapy of major and minor administrations along with conventional HF treatment for 5 weeks. Before and after ozone therapy, left ventricular end-systolic and end-diastolic volumes (LVESV, LVEDV) and the 6 minute walk distance (6MWD) and blood levels of the superoxide dismutase (SOD), catalase (CAT), glutathione (GSH), glutathione peroxidase (GSHPx), malondialdehyde (MDA), nitric oxide (NO) and N-terminal pro-brain natriuretic peptide (NTproBNP) were measured. Ozone therapy significantly reduced the serum levels of $N O$ and $M D A(P<0.001$, respectively) and signifi- cantly increased the levels of SOD, CAT, GSH and GSHPx $(P<0.001$, respectively). LVEDV and LVESV were found to be significantly reduced; however, $L V E F$ was not found to be significantly increased $(P=0.567)$. As the biochemical improvement marker of HF, NT-proBNP was significantly reduced $(P<0.001)$. The clinical HF improvement marker of 6 minute walk distance was also modestly increased $(P<0.001)$. Ozone therapy might be beneficial in terms of activating antioxidant system and merit further therapeutic potential to conventional HF treatment in patients with HFrEF.

Control case study on 80 patients in chronic heart failure NYHA 2 E 3 and FE $<35 \%$. In the study the patients received ozone by auto-hemoinfusion for five weeks in addition to the standard therapies for decompensation. Systolic and diastolic function and proto and tele systolic and diastolic volumes, six-minute walk test, blood levels of superoxide dismutase, catalase, glutathione, glutathione peroxidase, nitric oxide were evaluated. The oxygenozone therapy increases levels of superoxide dismutase, catalase glutathione, glutathione peroxidase, reduces the telesystolic and telediastolic volume of both ventricles and leads to modest improvements in six-minute walk testing.

Ozone therapy can therefore play an adjuvant role in chronic heart failure associated with standard therapies by activating and stimulating the natural antioxidant system.

A GP has a large number of patients with chronic heart failure: some of them, in agreement with their cardiologist, could benefit from combined ozone-conventional drug therapy. It should be noted that due to the chronicity of the disease it may be quite expensive to perform regular auto-hemotransfusions, so it should be considered carefully, in agreement with the patients.

- Effectiveness of a short-term treatment of oxygen-ozone therapy into healing in a posttraumatic wound, an Italian study of the University of Pavia by Degli Agosti et al. ${ }^{6}$

Abstract: Introduction. A number of studies suggest that oxygenozone therapy may have a role in the treatment of chronic, nonhealing, or ischemic wounds for its disinfectant and antibacterial properties. Nonhealing wounds are a significant cause of morbidity. Here we present a case of subcutaneous oxygen-ozone therapy used to treat a nonhealing postoperative wound in a young man during a period of 5 weeks. Case Presentation. A 46-year-old man had a motorcycle accident and underwent amputation of the right tibia and fibula. At the discharge he came to our attention to start rehabilitation treatment. At that time the wound was ulcerated but it was afebrile with no signs of inflammation and negativity to blood tests. At 2 months from the trauma despite appropriate treatment and dressing, the wound was slowly improving and the patient complained of pain. For this reason in addition to standard dressing he underwent oxygen-ozone therapy. After 5 weeks of treatment the wound had healed. Conclusion. In patients with nonhealing wounds, oxygen-ozone therapy could be helpful in speeding the healing and reducing the pain thanks to its disinfectant property and by the increase of endogenous oxygen free radicals'scavenging properties. Compared to standard dressing and other treatments reported in the literature it showed a shorter time of action.

Clinical case of a 46-year-old man with tibia-perone amputation from trauma in a road accident whose wound after two months of standard treatments struggled to heal, was then treated with subcutaneous injections of ozone and after 5 weeks the wound and ulcers closed. This clinical case suggested that subcutaneous ozone therapy near difficult wounds could help speed up healing times and reduce pain.

The GP interfaces with numerous wounds and skin ulcers that are difficult to heal: in selected cases, the addition of ozone therapy 
to standard therapies can be of great help to both the patient and the doctor because it involves no risk, is easy to apply and the cost is negligible.

- Cerebrovascular pattern improved by ozone autohemotherapy: an entropy-based study on Multiple Sclerosis patients, a multicentric study by Molinari et al. ${ }^{7}$

Abstract: Ozone major autohemotherapy is effective in reducing the symptoms of multiple sclerosis (MS) patients, but its effects on brain are still not clear. In this work, we have monitored the changes in the cerebrovascular pattern of MS patients and normal subjects during major ozone autohemotherapy by using near-infrared spectroscopy (NIRS) as functional and vascular technique. NIRS signals are analyzed using a combination of time, time-frequency analysis and nonlinear analysis of intrinsic mode function signals obtained from empirical mode decomposition technique. Our results show that there is an improvement in the cerebrovascular pattern of all subjects indicated by increasing the entropy of the NIRS signals. Hence, we can conclude that the ozone therapy increases the brain metabolism and helps to recover from the lower activity levels which is predominant in MS patients.

In this multicentre study (Italian and Japanese), the auto-hemoinfusion with oxygen-ozone was associated with standard therapies for multiple sclerosis (steroids and immunosuppressants). Through NIRS technique, it was demonstrated that ozone therapy increased brain metabolism and a subjective improvement of symptoms was achieved. There were no significant improvements in imaging or rheology.

This study highlighted the enormous potential that ozone can have in inflammatory diseases and their systemic complications when combined with standard therapies.

The GP does not deal directly with the therapies of multiple sclerosis patients, but plays a direct role in the management of complications, therapeutic advice and inter-specialist management, so knowledge of oxygen-ozonotherapy can be helpful for the GP who can advise additional evaluation by an ozonotherapist in selected cases.

\section{Conclusions}

Oxygen-ozone therapy is a clinical practice validated by numerous international studies for many diseases of different medical and surgical branches.

Studies show good efficacy, especially when used as an adjuvant to conventional therapies and good tolerability with very few and slight side effects and contraindications. Moreover, by combining it with standard therapies, it allows in many cases to reduce the overall dosages of drugs and the health spending for the National Health System.

Not being included in the National Health System in the Essential Levels of Care, the costs remain the full responsibility of the patient. In some types of treatment, such as the use of ozonized water (for H. pylori gastritis, mouth ulcers, oral diseases and chronic intestinal inflammatory diseases) the cost is very low; while in others, especially for chronic diseases (chronic heart failure, multiple sclerosis, systemic sclerosis, etc.) where a cycle of treatments is not sufficient but it is necessary to repeat the therapy in chronic, the cost is higher.

The GP has however the chance to know his/her patients in depth and may be able to assess which of those, among them who could benefit from the ozone therapy, can actually bear the costs of this therapy. Also in this case, the GP plays a key role in managing the pathologies of his/her patients and the resources that the Health System provides. By integrating standard therapies with ozone therapy, the GP can try to tailor the best combination of therapies for a given pathology for a given patient with the economic and cultural availability of the same, while fully respecting the therapeutic alliance.

In order to reduce the therapeutic costs, maximize the benefits and bring patients closer to the combination of standard therapies with ozone therapy, the GP can recommend Ozone Therapy centers or, after a SIOOT university master's degree, practise ozone therapy him/herself in his/her own practice.

\section{References}

1. Paoloni M, Di Sante L, Cacchio A, et al. Intramuscular oxygen-ozone therapy in the treatment of acute back pain with lumbar disc herniation: a multicenter, randomized, doubleblind, clinical trial of active and simulated lumbar paravertebral injection. Spine 2009;34:1337-44.

2. Fedorov AA, Gromov AS, Sapronenok SV, et al. Ozone therapy in gastroduodenal pathology associated with Helicobacter pylori. Vopr Kurortol Fizioter Lech Fiz Kult 2006;6:34-7.

3. Karatieieva S, Plesh I, Yurkiv O, et al. New method of treatment of pyoinflammatory soft tissue complications in patients with diabetes mellitus. Georgian Med News 2017;264:58-60.

4. Nowicka D. Thermography improves clinical assessment in patients with systemic sclerosis treated with ozone therapy. Biomed Res Int 2017;2017:5842723.

5. Buyuklu M, Kandemir FM, Set T, et al. Beneficial effects of ozone therapy on oxidative stress, cardiac functions and clinical findings in patients with heart failure reduced ejection fraction. Cardiac Toxicol 2017; 17:426-33.

6. Degli Agosti I, Ginelli E, Mazzacane B, et al. Effectiveness of a short-term treatment of oxygen-ozone therapy into healing in a posttraumatic wound. Case Rep Med 2016;2016:9528572.

7. Molinari F, Rimini D, Liboni W, et al. Cerebrovascular pattern improved by ozone autohemotherapy: an entropy-based study on multiple sclerosis patients. Med Biol Eng Comput 2017;55:1163-75.

8. Barbosa DC, Angelos JSD, Macena GMJ, et al. Effects of ozone on the pain and disability in patients with failed back surgery syndrome. Rev Assoc Med Bras (1992) 2017;63:355-60.

9. Braidy N, Izadi M, Sureda A, et al. Therapeutic relevance of ozone therapy in degenerative diseases: focus on diabetes and spinal pain. J Cell Physiol 2018;223:2705-14.

10. Molinari F, Simonetti V, Franzini M, et al. Ozone autohemotherapy induces long-term cerebral metabolic changes in multiple sclerosis patients. Int J Immunopathol Pharmacol 2014;27:379-89.

11. Bonforte G, Bellasi A, Riva H, et al. Ozone therapy: a potential adjunct approach to lower urinary tract infection? A case series report. G Ital Nefrol 2013;30:pii:gin/30.4.16.

12. Pandolfi S, Zammitti A, Franzini M, et al. Oxygen-ozone therapy in prevention and rehabilitation of myocardium infarct. Ozone Ther 2017;3:7512.

13. Remondino M, Valdenassi L, Franzini M. Pharmacoeconomic analysis of ozone therapy supported by agent based process simulation and data mining. Ozone Ther 2018;3:7518.

14. Valdenassi L, Franzini M, Simonetti V, Ricevuti G. Oxygenozone therapy: paradoxical stimulation of ozone. Ozone Therapy 2016;1:5837. 
15. Pandolfi S, Di Giovanni C, Marinari E, Franzini M. Improvement of neurologiocal conditions and recovery of the left ventricular ejection fraction subsequent to oxygen-ozone therapy through auto-hemoinfusion of ozonated blood. Ozone Ther 2016;1:5840.

16. Franzini M, Valdenassi L, Ionita G. First evaluations of oxygen-ozone therapy in antibiotic-resistant infections. Ozone Ther 2016;1:5838.

17. Della Puppa A, Franzini M. Short time recovery from severe knee algodistrophy treated with ozone-oxygen autohemotherapy. Ozone Ther 2016;1:6473.

18. Rimini D, Molinari F, Liboni W, et al. The speed of reinfusion affects the vascular system during ozone major autohemotherapy. Ozone Ther 2016;1:6477.

19. Balestrero R, Franzini M, Valdenassi L. Use of oxygen-ozone therapy in the treatment of fibromyalgia. Ozone Ther 2017;2:6744.

20. Giuliani G, Ricevuti G, Galoforo A, Franzini M. Microbiological aspects of ozone: bactericidal activity and antibiotic/antimicrobial resistance in bacterialstarins treated with ozone. Ozone Ther 2018;3:7971.

21. Bonforte G, Riva H, Giannini O, et al. Trattamento della sclerodermia con Ossigeno Ozono. Atti Congresso Internazionale SIOOT Ossigeno Ozono terapia, 16-18 marzo 2018. SIOOT; 2018. 